\title{
NONLINEAR VIBRATIONS OF FUNCTIONALLY GRADED CYLINDRICAL SHELLS: EFFECT OF THE GEOMETRY
}

\author{
Matteo Strozzi \\ Department of Mechanical and Civil Engineering \\ University of Modena and Reggio Emilia \\ 41121 Modena, Italy \\ matteo.strozzi@unimore.it
}

\author{
Francesco Pellicano \\ Antonio Zippo \\ Department of Mechanical and Civil Engineering \\ University of Modena and Reggio Emilia \\ 41121 Modena, Italy
}

\begin{abstract}
In this paper, the effect of the geometry on the nonlinear vibrations of functionally graded (FGM) cylindrical shells is analyzed. The Sanders-Koiter theory is applied to model the nonlinear dynamics of the system in the case of finite amplitude of vibration. The shell deformation is described in terms of longitudinal, circumferential and radial displacement fields. Simply supported boundary conditions are considered. The displacement fields are expanded by means of a double mixed series based on harmonic functions for the circumferential variable and Chebyshev polynomials for the longitudinal variable. In the linear analysis, after spatial discretization, mass and stiff matrices are computed, natural frequencies and mode shapes of the shell are obtained. In the nonlinear analysis, the three displacement fields are re-expanded by using approximate eigenfunctions obtained by the linear analysis; specific modes are selected. The Lagrange equations reduce nonlinear partial differential equations to a set of ordinary differential equations. Numerical analyses are carried out in order to characterize the nonlinear response of the shell. A convergence analysis is carried out to determine the correct number of the modes to be used. The analysis is focused on determining the nonlinear character of the response as the geometry of the shell varies.
\end{abstract}

\section{INTRODUCTION}

FGMs are composite materials obtained by combining different constituent materials, which are distributed along the thickness in accordance with a volume fraction law. The idea of FGMs was first introduced in $1984 / 87$ by a group of Japanese material scientists [1]. Loy et al. [2] analyzed the vibrations of FGM cylindrical shells considering simply supported boundary conditions. Leissa [3] studied the linear dynamics of shells with different topologies and materials. Yamaki [4] studied buckling and post-buckling of the shells in linear and nonlinear fields, reporting solution methods, numerical and experimental results. A modern treatise on the shells dynamics and stability can be found in Ref. [5], where also FGMs are analyzed. Pellicano et al. [6] considered the effect of the geometry on the nonlinear vibrations of homogeneous isotropic shells, leading to similar conclusions of the present work. The method of solution used in the present work was developed in Ref. [7]. In this paper, the effect of the geometry on the nonlinear vibrations of FGM cylindrical shells is analyzed; the Sanders-Koiter theory is applied to model the nonlinear dynamics of the system in the case of finite amplitude of vibration. The shell deformation is described in terms of longitudinal, circumferential and radial displacement fields. Simply supported boundary conditions are considered. The FGM is made of stainless steel and nickel, the material properties are graded along the thickness according to a volume fraction law. The solution method consists of two steps: 1) linear analysis and eigenfunctions evaluation; 2) nonlinear analysis, using an eigenfunction-based expansion. In the linear analysis, the displacement fields are expanded by means of a double mixed series based on harmonic functions for the circumferential variable and Chebyshev polynomials for the longitudinal variable. A Ritz based method allows to obtain approximate natural frequencies and mode shapes. In the nonlinear analysis, the three displacement fields are reexpanded by using the approximate eigenfunctions; an energy approach based on Lagrange equations is considered to reduce the nonlinear partial differential equations to a set of nonlinear ordinary differential equations. Numerical analyses are carried out in order to characterize the nonlinear response when the shell is subjected to an harmonic external load; a convergence analysis is carried out in order to obtain the correct number of the axisymmetric and asymmetric modes. The effect of the geometry on the nonlinear vibrations of the shells is analyzed, and a comparison of nonlinear amplitude-frequency curves of the FGM shells with different geometries is carried out. 


\section{FUNCTIONALLY GRADED MATERIALS}

A generic material property $P_{f g m}$ of a FGM depends on the material properties and the volume fractions of the constituent materials, it is expressed in the form [2]

$$
P_{f g m}(T, z)=\sum_{i=1}^{k} \tilde{P}_{i}(T) V_{f i}(z)
$$

where $\tilde{P}_{i}$ and $V_{f i}$ are the material property and the volume fraction of the constituent material $i$. The material property $\tilde{P}_{i}$ of a constituent material can be described as a function of the environmental temperature $T(\mathrm{~K})$ by Touloukian's relation [2] (the index $i$ is dropped for the sake of simplicity)

$$
\tilde{P}(T)=P_{0}\left(P_{-1} T^{-1}+1+P_{1} T+P_{2} T^{2}+P_{3} T^{3}\right)
$$

where $P_{0}, P_{-1}, P_{1}, P_{2}$ and $P_{3}$ are the coefficients of temperature of the constituent material. In the case of a FGM thin circular cylindrical shell with a uniform thickness $h$ and a reference surface at its middle surface, the volume fraction $V_{f}$ of a constituent material can be written as [2]

$$
V_{f}(z)=\left(\frac{z+h / 2}{h}\right)^{p}
$$

where the power-law exponent $p$ is a positive real number, $(0 \leq p \leq \infty)$, and $z$ describes the radial distance measured from the middle surface of the shell, $(-h / 2 \leq z \leq h / 2)$, see Fig. 1. For a FGM thin cylindrical shell made of two different constituent materials, the volume fractions $V_{f 1}$ and $V_{f 2}$ can be written in the following form [2]

$$
V_{f 1}(z)=1-\left(\frac{z+h / 2}{h}\right)^{p} \quad V_{f 2}(z)=\left(\frac{z+h / 2}{h}\right)^{p}
$$

Young's modulus $E$, Poisson's ratio $v$ and mass density $\rho$ are expressed as [2]

$$
\begin{aligned}
& E_{f g m}(T, z)=\left(E_{2}(T)-E_{1}(T)\right)\left(\frac{z+h / 2}{h}\right)^{p}+E_{1}(T) \\
& v_{f g m}(T, z)=\left(v_{2}(T)-v_{1}(T)\right)\left(\frac{z+h / 2}{h}\right)^{p}+v_{1}(T) \\
& \rho_{f g m}(T, z)=\left(\rho_{2}(T)-\rho_{1}(T)\right)\left(\frac{z+h / 2}{h}\right)^{p}+\rho_{1}(T)
\end{aligned}
$$

\section{SANDERS-KOITER NONLINEAR THEORY OF SHELLS}

In Figure 1, a FGM circular cylindrical shell having radius $R$, length $L$ and thickness $h$ is shown; a cylindrical coordinate system $(0 ; x, \theta, z)$ is considered in order to take advantage from the axial symmetry of the structure, the origin $O$ of the reference system is located at the center of one end of the shell. Three displacement fields are represented in Fig. 1: longitudinal $u(x, \theta, t)$, circumferential $v(x, \theta, t)$ and radial $w(x, \theta, t)$.

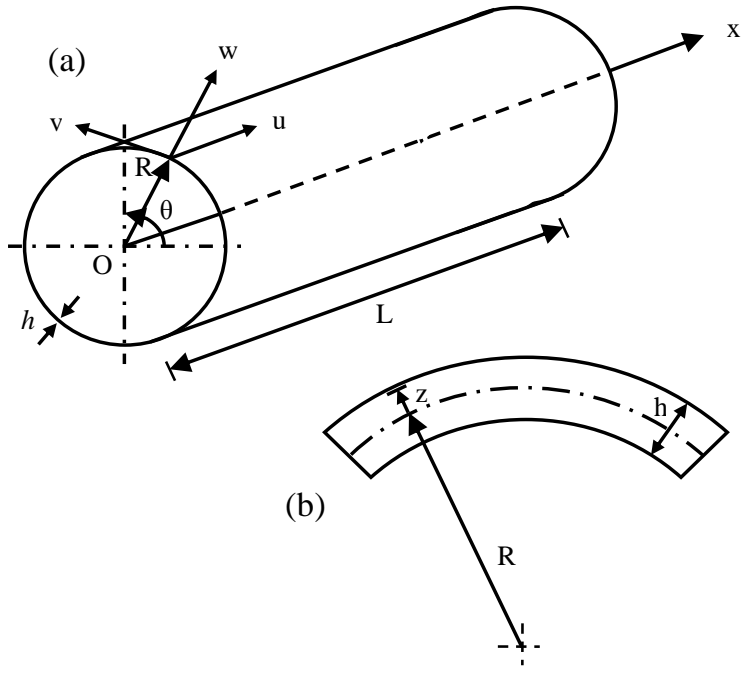

Figure 1. GEOMETRY OF THE FGM CYLINDRICAL SHELL. (a) COMPLETE SHELL; (b) CROSS-SECTION OF THE SHELL SURFACE.

The Sanders-Koiter nonlinear theory of shells is an eight-order theory based on the Love's "first approximation" [3]. The strain components $\left(\varepsilon_{x}, \varepsilon_{\theta}, \gamma_{x \theta}\right)$ at an arbitrary point of the shell are related to the middle surface strains $\left(\varepsilon_{x, 0}, \varepsilon_{\theta, 0}, \gamma_{x \theta, 0}\right)$ and to the changes in the curvature and torsion $\left(k_{x}, k_{\theta}, k_{x \theta}\right)$ of the middle surface of the shell by the following relationships [4]

$$
\varepsilon_{x}=\varepsilon_{x, 0}+z k_{x} \quad \varepsilon_{\theta}=\varepsilon_{\theta, 0}+z k_{\theta} \quad \gamma_{x \theta}=\gamma_{x \theta, 0}+z k_{x \theta}
$$

where $z$ is the distance of the arbitrary point of the cylindrical shell from the middle surface and $(x, \theta)$ are the longitudinal and angular coordinates of the shell, see Fig. 1. The middle surface strains and changes in curvature and torsion are given by [4]

$$
\begin{gathered}
\varepsilon_{x, 0}=\frac{\partial u}{L \partial \eta}+\frac{1}{2}\left(\frac{\partial w}{L \partial \eta}\right)^{2}+\frac{1}{8}\left(\frac{\partial v}{L \partial \eta}-\frac{\partial u}{R \partial \theta}\right)^{2} \\
\varepsilon_{\theta, 0}=\frac{\partial v}{R \partial \theta}+\frac{w}{R}+\frac{1}{2}\left(\frac{\partial w}{R \partial \theta}-\frac{v}{R}\right)^{2}+\frac{1}{8}\left(\frac{\partial u}{R \partial \theta}-\frac{\partial v}{L \partial \eta}\right)^{2} \\
\gamma_{x \theta, 0}=\frac{\partial u}{R \partial \theta}+\frac{\partial v}{L \partial \eta}+\frac{\partial w}{L \partial \eta}\left(\frac{\partial w}{R \partial \theta}-\frac{v}{R}\right) \\
k_{x}=-\frac{\partial^{2} w}{L^{2} \partial \eta^{2}} \quad k_{\theta}=\frac{\partial v}{R^{2} \partial \theta}-\frac{\partial^{2} w}{R^{2} \partial \theta^{2}} \\
k_{x \theta}=-2 \frac{\partial^{2} w}{L R \partial \eta \partial \theta}+\frac{1}{2 R}\left(3 \frac{\partial v}{L \partial \eta}-\frac{\partial u}{R \partial \theta}\right)
\end{gathered}
$$

where $(\eta=x / L)$ represents the nondimensional longitudinal coordinate. 
In the case of FGMs, the stresses are related to the strains as follows [5]

$$
\begin{gathered}
\sigma_{x}=\frac{E(z)}{1-v^{2}(z)}\left(\varepsilon_{x}+v(z) \varepsilon_{\theta}\right) \quad \sigma_{\theta}=\frac{E(z)}{1-v^{2}(z)}\left(\varepsilon_{\theta}+v(z) \varepsilon_{x}\right) \\
\tau_{x \theta}=\frac{E(z)}{2(1+v(z))} \gamma_{x \theta}
\end{gathered}
$$

where $E(z)$ is the Young's modulus and $v(z)$ is the Poisson's ratio $\left(\sigma_{z}=0\right.$, plane stress hypotheses).

The elastic strain energy $U$ of a cylindrical shell is given by [5]

$$
U=\frac{1}{2} L R \int_{0}^{1} \int_{0}^{2 \pi} \int_{-h / 2}^{h / 2}\left(\sigma_{x} \varepsilon_{x}+\sigma_{\theta} \varepsilon_{\theta}+\tau_{x \theta} \gamma_{x \theta}\right) d \eta d \theta d z
$$

The kinetic energy $T$ of a cylindrical shell (rotary inertia effect is neglected) is given by [5]

$$
T=\frac{1}{2} L R \int_{0}^{1} \int_{0}^{2 \pi} \int_{-h / 2}^{h / 2} \rho(z)\left(\dot{u}^{2}+\dot{v}^{2}+\dot{w}^{2}\right) d \eta d \theta d z
$$

where $\rho(z)$ is the mass density of the shell.

The virtual work $W$ done by the external forces is written as [5]

$$
W=L R \int_{0}^{1} \int_{0}^{2 \pi}\left(q_{x} u+q_{\theta} v+q_{z} w\right) d \eta d \theta
$$

where $\left(q_{x}, q_{\theta}, q_{z}\right)$ are the distributed forces per unit area acting in longitudinal, circumferential and radial direction.

The nonconservative damping forces are assumed to be of viscous type and are taken into account by using Rayleigh's dissipation function (viscous damping coefficient $c$ ) [5]

$$
F=\frac{1}{2} c L R \int_{0}^{2 \pi} \int_{0}^{1}\left(\dot{u}^{2}+\dot{v}^{2}+\dot{w}^{2}\right) d \eta d \theta
$$

\section{VIBRATION ANALYSIS}

In order to carry out the dynamic analysis of the shell, a two-steps procedure is considered [7]: $i$ ) the Rayleigh-Ritz method is applied to the linearized formulation of the problem, in order to obtain an approximation of the eigenfunctions; ii) the displacement fields are re-expanded using the approximate eigenfunctions, the Lagrange equations are considered in conjunction with the fully nonlinear expression of the potential energy, in order to obtain a set of nonlinear ordinary differential equations in modal coordinates.

\section{Linear Vibration Analysis}

In order to carry out a linear vibration analysis, only the quadratic terms are retained in Eqn. (11). A modal vibration, i.e., a synchronous motion, can be written in the form [7]

$$
\begin{gathered}
u(\eta, \theta, t)=U(\eta, \theta) f(t) \quad v(\eta, \theta, t)=V(\eta, \theta) f(t) \\
w(\eta, \theta, t)=W(\eta, \theta) f(t)
\end{gathered}
$$

where $u(\eta, \theta, t), v(\eta, \theta, t), w(\eta, \theta, t)$ describe the displacement fields, $U(\eta, \theta), V(\eta, \theta), W(\eta, \theta)$ represent the modal shape and $f(t)$ describes the time law, which is supposed to be the same for each displacement field (synchronous motion hypothesis).

The components of the modal shape are expanded by means of a double mixed series: the periodicity of deformation in the circumferential direction suggests the use of harmonic functions $(\cos n \theta, \sin n \theta)$, Chebyshev orthogonal polynomials are considered in the longitudinal direction $T_{m}^{*}(\eta)$ [7]

$$
\begin{aligned}
& U(\eta, \theta)=\sum_{m=0}^{M_{u}} \sum_{n=0}^{N} \widetilde{U}_{m, n} T_{m}^{*}(\eta) \cos n \theta \\
& V(\eta, \theta)=\sum_{m=0}^{M_{v}} \sum_{n=0}^{N} \widetilde{V}_{m, n} T_{m}^{*}(\eta) \sin n \theta \\
& W(\eta, \theta)=\sum_{m=0}^{M_{W}} \sum_{n=0}^{N} \widetilde{W}_{m, n} T_{m}^{*}(\eta) \cos n \theta
\end{aligned}
$$

where $T_{m}^{*}(\eta)=T_{m}(2 \eta-1), m$ is the number of longitudinal half-waves, $n$ is the number of nodal diameters and $\left(\widetilde{U}_{m, n}, \widetilde{V}_{m, n}, \widetilde{W}_{m, n}\right)$ are the generalized coordinates.

\section{Boundary Conditions}

Simply supported boundary conditions are given by [3]

$$
w=0 \quad v=0 \quad M_{x}=0 \quad N_{x}=0 \quad \text { for } \eta=0,1
$$

where forces and moments are given by [3]

$$
N_{x}=\frac{E h}{1-v^{2}}\left(\varepsilon_{x, 0}+v \varepsilon_{\theta, 0}\right) \quad M_{x}=\frac{E h^{3}}{12\left(1-v^{2}\right)}\left(k_{x}+v k_{\theta}\right)
$$

The previous conditions imply the following equations [7]

$$
\begin{array}{llll}
\sum_{m=0}^{M_{w}} \widetilde{W}_{m, n} T_{m}^{*}(\eta)=0 & \theta \in[0,2 \pi] & n \in[0, N] & \text { for } \eta=0,1 \\
\sum_{m=0}^{M_{v}} \tilde{V}_{m, n} T_{m}^{*}(\eta)=0 & \theta \epsilon[0,2 \pi] & n \in[0, N] & \text { for } \eta=0,1 \\
\sum_{m=0}^{M_{w}} \widetilde{W}_{m, n} T_{m, \eta \eta}^{*}(\eta)=0 & \theta \epsilon[0,2 \pi] & n \in[0, N] & \text { for } \eta=0,1 \\
\sum_{m=0}^{M_{u}} \widetilde{U}_{m, n} T_{m, \eta}^{*}(\eta)=0 & \theta \epsilon[0,2 \pi] & n \in[0, N] & \text { for } \eta=0,1
\end{array}
$$

The linear algebraic system given by Eqns. $(19-22)$ can be solved analytically in terms of the coefficients $\left(\widetilde{U}_{1, n}, \widetilde{U}_{2, n}, \widetilde{V}_{0, n}\right.$, $\left.\widetilde{V}_{1, n}, \widetilde{W}_{0, n}, \widetilde{W}_{1, n}, \widetilde{W}_{2, n}, \widetilde{W}_{3, n}\right)$, for $n \epsilon[0, N]$; such coefficients are linearly dependent from the others. 


\section{Rayleigh-Ritz Procedure}

The maximum number of variables needed for describing a generic vibration mode is obtained by the following relation $\left(N_{p}=M_{u}+M_{v}+M_{w}-5\right)$, where $M_{u}=M_{v}=M_{w}$ represent the maximum degree of the Chebyshev polynomials.

For a multi-mode analysis including different nodal diameters $n$, the number of degrees of freedom of the system is computed by the relation $\left(N_{\max }=N_{p} \times(N+1)\right)$, where $N$ describes the maximum number of nodal diameters. The equations (15) are inserted into the expressions of $U$ and $T$ (Eqns. $(11-12)$ ), in order to develop the Rayleigh quotient $R(\widetilde{\mathbf{q}})=\frac{U_{\max }}{T^{*}}$, where $U_{\max }=\max (U)$ is the maximum of the elastic strain energy during a modal vibration, $T^{*}=\frac{T_{\max }}{\omega^{2}}, T_{\max }=\max (T)$ is the maximum of the kinetic energy, $\omega$ is the circular frequency of the harmonic motion and $\widetilde{\mathbf{q}}=\left[\ldots, \widetilde{U}_{m, n}, \widetilde{V}_{m, n}, \widetilde{W}_{m, n}, \ldots\right]^{T}$ is a vector containing all the unknowns. After imposing the stationarity to the Rayleigh quotient, one obtains the eigenvalue problem [7]

$$
\left(-\omega^{2} \mathbf{M}+\mathbf{K}\right) \widetilde{\mathbf{q}}=\mathbf{0}
$$

which furnishes natural frequencies and modes of vibration (eigenvalues and eigenvectors). The modal shape is given by the Eqns. (16), where the coefficients $\left(\widetilde{U}_{m, n}, \widetilde{V}_{m, n}, \widetilde{W}_{m, n}\right)$ are substituted with $\left(\widetilde{U}_{m, n}^{(j)}, \widetilde{V}_{m, n}^{(j)}, \widetilde{W}_{m, n}^{(j)}\right)$, which are the components of the $\mathrm{j}$-th eigenvector $\widetilde{\mathbf{q}}_{\mathbf{j}}$ of equation (23). The vector function $\boldsymbol{U}^{(j)}(\eta, \theta)=\left[U^{(j)}(\eta, \theta), V^{(j)}(\eta, \theta), W^{(j)}(\eta, \theta)\right]^{T}$ represents an approximation of the $\mathrm{j}$-th mode of the original problem; the eigenfunctions are then eventually normalized by imposing $\max \left[\max \left[U^{(j)}(\eta, \theta)\right], \max \left[V^{(j)}(\eta, \theta)\right], \max \left[W^{(j)}(\eta, \theta)\right]\right]=$

1 , see Ref. [7] for explanation.

\section{Nonlinear Vibration Analysis}

In the nonlinear vibration analysis, the full expression of the elastic strain energy (11), containing terms up to the fourth order (cubic nonlinearity), is considered. The displacement fields $u(\eta, \theta, t), v(\eta, \theta, t), w(\eta, \theta, t)$ are expanded by using the linear mode shapes $U(\eta, \theta), V(\eta, \theta), W(\eta, \theta)$ obtained in the previous section [7]

$$
\begin{gathered}
u(\eta, \theta, t)=\sum_{j=1}^{N_{u}} U^{(j)}(\eta, \theta) f_{u, j}(t) \\
v(\eta, \theta, t)=\sum_{j=1}^{N_{v}} V^{(j)}(\eta, \theta) f_{v, j}(t) \\
w(\eta, \theta, t)=\sum_{j=1}^{N_{w}} W^{(j)}(\eta, \theta) f_{w, j}(t)
\end{gathered}
$$

These expansions respect exactly the boundary conditions; the synchronicity is relaxed as for each mode and each component $(u, v, w)$ different time laws are allowed. The mode shapes $U^{(j)}(\eta, \theta), V^{(j)}(\eta, \theta), W^{(j)}(\eta, \theta)$ are known functions, they are expressed in terms of polynomials and harmonic functions. The Lagrange equations are expressed in the following form [7]

$$
\frac{d}{d t}\left(\frac{\partial L}{\partial \dot{q}_{i}}\right)-\frac{\partial L}{\partial q_{i}}=Q_{i} \quad \text { for } i \epsilon\left[1, N_{\max }\right] \quad\left(L=T_{s}-U_{s}\right)
$$

where the modal coordinates are now ordered in a vector $\mathbf{q}(t)=\left[\ldots f_{u, j}, f_{v, j}, f_{w, j}, \ldots\right]$, and $N_{\max }$ depends on the number of modes considered in the expansions (24). The generalized forces $Q_{i}$ are obtained by the differentiation of the Rayleigh's dissipation function $F(14)$ and the virtual work of the external forces $W$ (13), in the form [7]

$$
Q_{i}=-\frac{\partial F}{\partial \dot{q}_{i}}+\frac{\partial W}{\partial q_{i}}
$$

Expansions (24) are inserted into strain energy (11), kinetic energy (12), virtual work of the external forces (13) and damping forces (14); using Lagrange Eqns. (25), a set of nonlinear ordinary differential equations (ODE) is obtained.

\section{NUMERICAL RESULTS}

In this section, the nonlinear vibrations of FGM shells with different modal shape expansions and geometries are analyzed. Analyses are carried out on a FGM made of stainless steel and nickel, its properties are graded along the thickness according to a volume fraction distribution, where $p$ is the power-law exponent. The material properties are reported in Tab. 1-2 [2].

Table 1. PROPERTIES OF STAINLESS STEEL VS. COEFFICIENTS OF TEMPERATURE.

\begin{tabular}{|c|c|c|c|}
\hline & \multicolumn{3}{|c|}{ stainless steel } \\
\hline & $\mathrm{E}$ & $v$ & $\rho$ \\
\hline $\mathrm{P}_{0}$ & $2.01 \times 10^{11} \mathrm{Nm}^{-2}$ & 0.326 & $8166 \mathrm{kgm}^{-3}$ \\
\hline $\mathrm{P}_{-1}$ & $0 \mathrm{~K}$ & $0 \mathrm{~K}$ & $0 \mathrm{~K}$ \\
\hline $\mathrm{P}_{1}$ & $3.08 \times 10^{-4} \mathrm{~K}^{-1}$ & $-2.002 \times 10^{-4} \mathrm{~K}^{-1}$ & $0 \mathrm{~K}^{-1}$ \\
\hline $\mathrm{P}_{2}$ & $-6.53 \times 10^{-7} \mathrm{~K}^{-2}$ & $3.797 \times 10^{-7} \mathrm{~K}^{-2}$ & $0 \mathrm{~K}^{-2}$ \\
\hline $\mathrm{P}_{3}$ & $0 \mathrm{~K}^{-3}$ & $0 \mathrm{~K}^{-3}$ & $0 \mathrm{~K}^{-3}$ \\
\hline $\mathrm{P}(300 \mathrm{~K})$ & $2.08 \times 10^{11} \mathrm{Nm}^{-2}$ & 0.318 & $8166 \mathrm{kgm}^{-3}$ \\
\hline
\end{tabular}

Table 2. PROPERTIES OF NICKEL VS. COEFFICIENTS OF TEMPERATURE.

\begin{tabular}{|c|c|c|c|}
\hline & \multicolumn{3}{|c|}{ nickel } \\
\hline & $\mathrm{E}$ & $v$ & $\rho$ \\
\hline $\mathrm{P}_{0}$ & $2.24 \times 10^{11} \mathrm{Nm}^{-2}$ & 0.310 & $8900 \mathrm{kgm}^{-3}$ \\
\hline $\mathrm{P}_{-1}$ & $0 \mathrm{~K}$ & $0 \mathrm{~K}$ & $0 \mathrm{~K}$ \\
\hline $\mathrm{P}_{1}$ & $-2.79 \times 10^{-4} \mathrm{~K}^{-1}$ & $0 \mathrm{~K}^{-1}$ & $0 \mathrm{~K}^{-1}$ \\
\hline $\mathrm{P}_{2}$ & $-3.99 \times 10^{-9} \mathrm{~K}^{-2}$ & $0 \mathrm{~K}^{-2}$ & $0 \mathrm{~K}^{-2}$ \\
\hline $\mathrm{P}_{3}$ & $0 \mathrm{~K}^{-3}$ & $0 \mathrm{~K}^{-3}$ & $0 \mathrm{~K}^{-3}$ \\
\hline $\mathrm{P}(300 \mathrm{~K})$ & $2.05 \times 10^{11} \mathrm{Nm}^{-2}$ & 0.310 & $8900 \mathrm{kgm}^{-3}$ \\
\hline
\end{tabular}




\section{Nonlinear Response Convergence Analysis}

The convergence analysis is carried out on a simply supported shell excited with an harmonic force; the excitation frequency is close to mode $(m, n)$. The convergence is checked by adding suitable modes to the resonant one, i.e., asymmetric modes $(k \times m, j \times n) k=1,3 j=1,2,3$ due to the presence of the quadratic and the cubic nonlinearities; axisymmetric modes $(k, 0) k=1,3,5,7$ due to the quadratic nonlinearities.

The convergence analysis is then developed by introducing a different number of asymmetric and axisymmetric modes in the expansions of the displacement fields $u, v, w$, see Tab. 3 .

Table 3. MODAL EXPANSION FOR THE NONLINEAR ANALYSIS.

\begin{tabular}{|c|l|c|c|c|c|c|c|c|c|c|}
\hline$(m, n)$ & $(1,6)$ & $(1,12)$ & $(1,18)$ & $(3,6)$ & $(3,12)$ & $(3,18)$ & $(1,0)$ & $(3,0)$ & $(5,0)$ & $(7,0)$ \\
\hline 6 dof & $u, v, w$ & $v$ & - & - & - & - & $u, w$ & - & - & - \\
\hline 9 dof & $u, v, w$ & $v$ & - & - & $v$ & - & $u, w$ & $u, w$ & - & - \\
\hline 12 dof & $u, v, w$ & $v$ & - & $u, v, w$ & $v$ & - & $u, w$ & $u, w$ & - & - \\
\hline 15 dof & $u, v, w$ & $v$ & $v$ & $u, v, w$ & $v$ & - & $u, w$ & $u, w$ & $u, w$ & - \\
\hline 18 dof & $u, v, w$ & $v$ & $v$ & $u, v, w$ & $v$ & $v$ & $u, w$ & $u, w$ & $u, w$ & $u, w$ \\
\hline
\end{tabular}

The FGM cylindrical shell is excited by means of an external modally distributed radial force $q_{z}=f_{1,6} \sin \eta \cos 6 \theta \cos \Omega t$; the amplitude of excitation is $f_{1,6}=0.0012 h^{2} \varrho \omega_{1,6}^{2}$ and the frequency of excitation $\Omega$ is close to the mode $(1,6), \Omega \cong \omega_{1,6}$. The external forcing $f_{1,6}$ is normalized with respect to mass, acceleration and thickness; the damping ratio is equal to $\xi_{1,6}=0.0005$. The nonlinear amplitudes $f_{u, 1}, f_{v, 1}, f_{w, 1}$ of the expansions (24) refer to the displacement fields $u, v, w$ of the mode $(1,6)$, respectively.

In Figure 2, a comparison of nonlinear amplitude-frequency curves of the cylindrical shell $(h / R=0.002, L / R=20, p=1)$ with different nonlinear expansions is shown; the shell is very thin and long. The nonlinear 6 dof model describes a wrong softening nonlinear behaviour, while the higher-order nonlinear expansions converge to a hardening nonlinear behaviour.

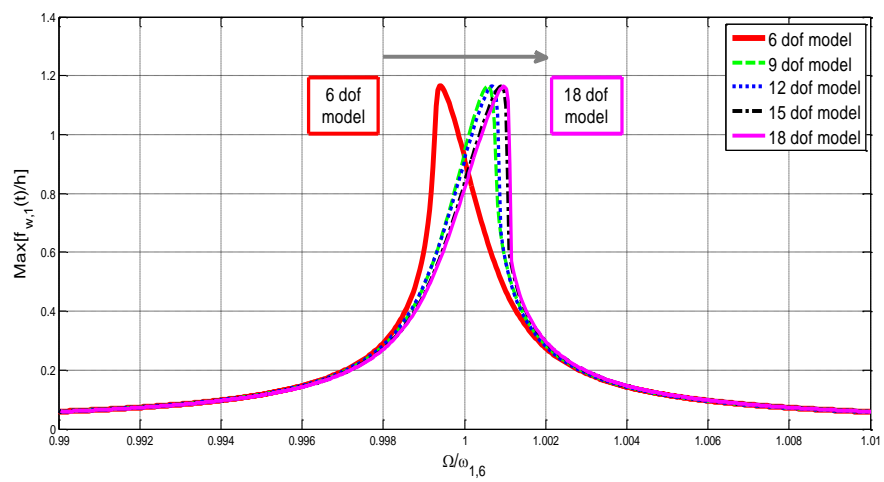

Figure 2. COMPARISON OF NONLINEAR AMPLITUDE-FREQUENCY CURVES OF THE FGM CYLINDRICAL SHELL ( $\mathrm{h} / \mathrm{R}=0.002, \mathrm{~L} / \mathrm{R}=20, \mathrm{p}=1$ ) “๓”, 6 DOF MODEL; “- - ", 9 DOF MODEL; “...”, 12 DOF MODEL; “- - -", 15 DOF MODEL; “-”, 18 DOF MODEL.

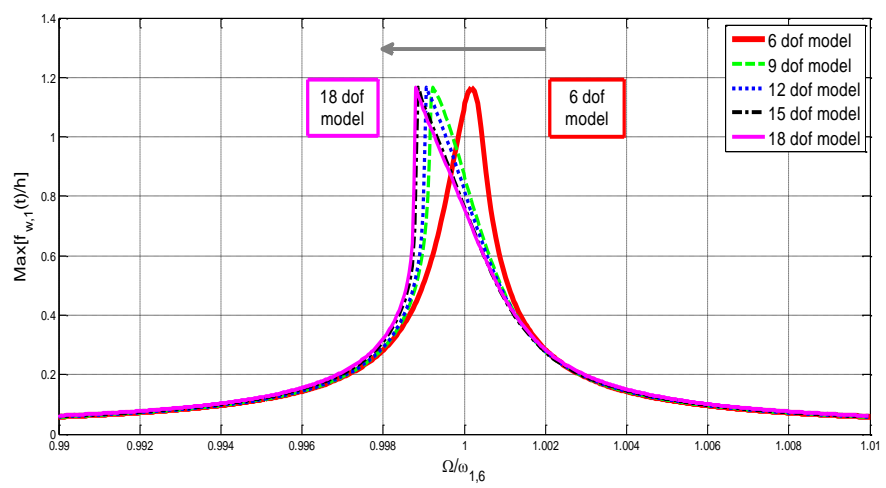

Figure 3. COMPARISON OF NONLINEAR AMPLITUDE-FREQUENCY CURVES OF THE FGM CYLINDRICAL SHELL $(h / R=0.025, L / R=20, p=1)$. “ø”, 6 DOF MODEL; “- - ”, 9 DOF MODEL; “...”, 12 DOF MODEL; “- ·-", 15 DOF MODEL; “-”, 18 DOF MODEL.

In Figure 3, a comparison of nonlinear amplitude-frequency curves of the cylindrical shell $(h / R=0.025, L / R=20, p=1)$ with different nonlinear expansions is shown; the shell is quite thick and long. The nonlinear 6 dof model describes a wrong hardening nonlinear behaviour, and the higher-order nonlinear expansions converge to a softening nonlinear behaviour.

In Figure 4, a comparison of nonlinear amplitude-frequency curves of the cylindrical shell $(h / R=0.050, L / R=20, p=1)$ with different nonlinear expansions is shown; the shell is thick and long. The nonlinear 6 dof model describes a wrong softening nonlinear behaviour, while the higher-order nonlinear expansions converge to a hardening nonlinear behaviour.

The fundamental role of the axisymmetric and higher-order asymmetric modes is clarified in order to obtain the actual character of the shell nonlinearity.

From the convergence analysis, it can be observed that the 9 dof model gives satisfactory results with the minimal computational effort; therefore, in the following analyses the 9 dof model will be used.

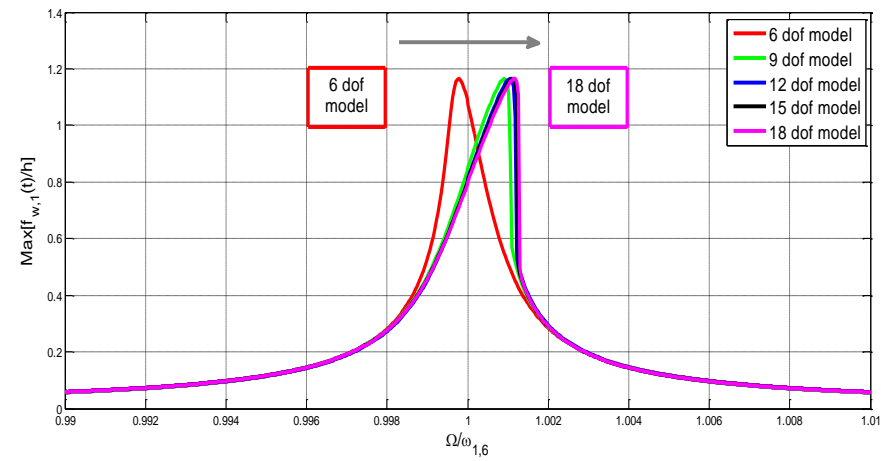

Figure 4. COMPARISON OF NONLINEAR AMPLITUDE-FREQUENCY CURVES OF THE FGM CYLINDRICAL SHELL $(\mathrm{h} / \mathrm{R}=0.050, \mathrm{~L} / \mathrm{R}=20, \mathrm{p}=1)$. “ロ”, 6 DOF MODEL; “" - ", 9 DOF MODEL; “...", 12 DOF MODEL; “- - -", 15 DOF MODEL; “-”, 18 DOF MODEL. 
In particular, the following 9 dof model will be considered for studying a generic resonant mode $(m, n)$ :

- $\quad$ modes $(m, n),(1,0),(3,0)$ for the field $u$

- $\quad \operatorname{modes}(m, n),(m, 2 n),(3 m, 2 n)$ for the field $v$

- $\quad \operatorname{modes}(m, n),(1,0),(3,0)$ for the field $w$

After selecting such modes, each expansion present in the Eqns. (24) is reduced to a three-terms modal expansion; the resulting nonlinear system has 9 dof.

The expression of the resulting discretized nonlinear equations of motion and the method used to compute the nonlinear response amplitude curves are described in Ref. [7].

\section{Effect of the Geometry}

In this part, the role of the geometric parameters $h, L, R$ and in particular their ratios $h / R$ and $L / R$ on the nonlinear response of the FGM cylindrical shells is clarified.

In Figure 5, a comparison of nonlinear amplitude-frequency curves of simply supported FGM circular shells with different geometries is shown: this figure represents the maximum of the modal amplitude $f_{w, 1}(t)$ of the mode $(1,6)$ (normalized by the thickness $h$ of the shell) versus the frequency of the excitation $\Omega$ (normalized by the frequency $\omega_{1,6}$ of the driven mode).

The nonlinear response is:

- $\quad$ hardening for $(h / R=0.002, L / R=20, p=1)$, red line $(-)$

- $\quad$ softening for $(h / R=0.025, L / R=20, p=1)$, black line (-)

- $\quad$ hardening for $(h / R=0.050, L / R=20, p=1)$, blue line $(-)$

The nonlinear response of the thicker circular cylindrical shell $(h / R=0.050, L / R=20, p=1)$ is more hardening than the thinner one $(h / R=0.002, L / R=20, p=1)$, a wide interval of thickness gives rise to softening type behaviour.

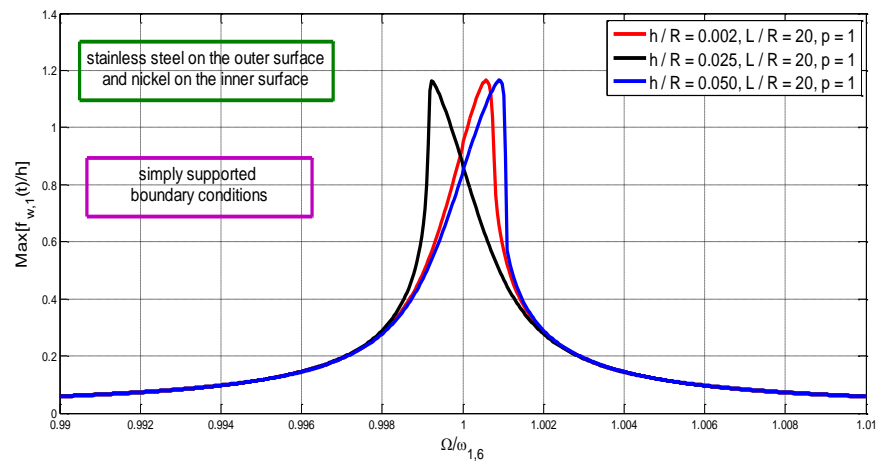

Figure 5. AMPLITUDE-FREQUENCY CURVES, EFFECT OF THICKNESS, 9 DOF MODEL, ASYMMETRIC MODE $(1,6)$. “-”, (h/R = 0.002, L/R = 20, p = 1); “-”, (h/R = 0.025, L/R = 20, p = 1); “-”, (h/R = 0.050, L/R = 20, p = 1).

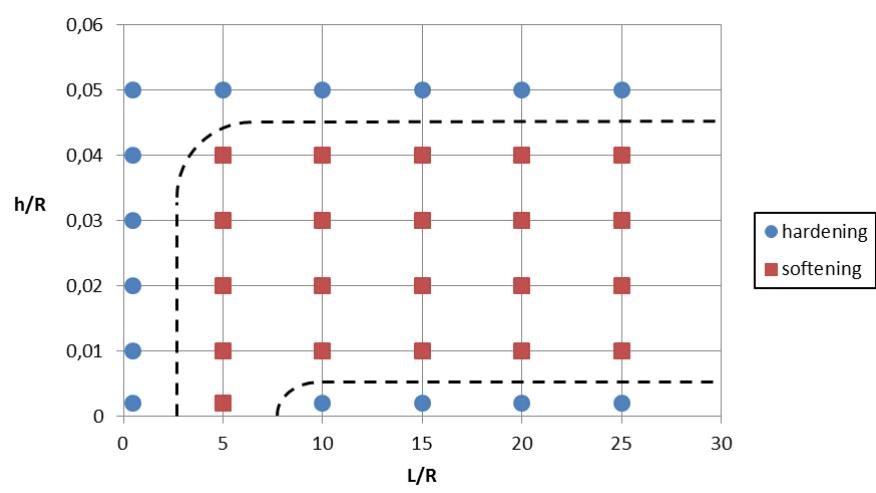

Figure 6. EFFECT OF THE GEOMETRY ON THE NONLINEAR RESPONSE OF THE FGM SHELL. CIRCLE BLUE MARKS: HARDENING; SQUARE RED MARKS: SOFTENING; DASHED LINES: BOUNDARIES BETWEEN HARDENING AND SOFTENING REGIONS (FROM REF. [6], HOMOGENEOUS MATERIALS).

In order to determine the influence of the geometry on the nonlinear vibration, a parametric analysis is carried out by varying the fundamental ratios $(h / R)$ and $(L / R)$.

In Figure 6, the effect of the geometry on the nonlinearity type is analyzed considering few numerical test-cases: square marks describe a softening behaviour, while circle marks describe a hardening behaviour. In Figure 6, the dashed lines, which are referred to homogeneous shells, are reproduced from Ref. [6]: such lines represent the boundaries of the hardening/softening regions. The present analysis shows that the FGM shells behave similarly to homogeneous ones: very short shells $(L / R<0.5)$ and thick shells $(h / R>0.045)$ present a hardening nonlinear behaviour; conversely, a softening nonlinearity is found in a wide range of shell geometries. However, for sufficiently long $(L / R>5)$ and thin $(h / R<0.005)$ shells, the system can be hardening again. This confirms for the FGM shells the results available in literature concerning homogeneous isotropic shells.

\section{CONCLUSIONS}

In this paper, the effect of the geometry on the nonlinear vibrations of FGM cylindrical shells is analyzed.

The Sanders-Koiter theory is applied to model the nonlinear dynamics of the system in the case of finite amplitude of vibration.

The functionally graded material is made of stainless steel and nickel, the material properties are graded along the thickness according to a volume fraction law.

Numerical analyses are carried out in order to characterize the nonlinear response when the shell is subjected to a harmonic external load.

A convergence analysis is carried out by introducing in the longitudinal, circumferential and radial displacement fields a different number of asymmetric and axisymmetric modes; the role of the axisymmetric and higher-order asymmetric modes is clarified in order to obtain the actual character of the shell nonlinearity. 
The effect of the geometry on the nonlinear vibrations of the FGM shells is analyzed: very short shells and thick shells show a hardening nonlinearity, conversely, a softening nonlinearity is found in a wide range of the shell geometries. For sufficiently long and thin shells, the system behaves in a hardening nonlinear way.

This paper confirms regarding the effect of the geometry on the nonlinear vibrations of FGM shells the results available in the literature concerning the nonlinear vibrations of homogeneous isotropic shells.

The same comparison between the nonlinear vibrations of isotropic and FGM shells have to be extended in future works to the effect of the boundary conditions, the distribution of the material and the participation of the companion modes.

\section{REFERENCES}

[1] Techreport, Japanese Government, 1987. Research on the basic technology for the development of functionally gradient materials for the relaxation of the thermal stress. Science and Technology Agency of Japan.

[2] Loy, C.T., Lam, K.Y., and Reddy, J.N., 1999. "Vibration of functionally graded cylindrical shells". International Journal of Mechanical Sciences, 41, pp. 309-324.

[3] Leissa, A.W., 1973. Vibrations of Shells. The Government Printing Office, Washington DC.

[4] Yamaki, N., 1984. Elastic Stability of Circular Cylindrical Shells. North-Holland, Amsterdam.

[5] Amabili, M., 2008. Nonlinear Vibrations and Stability of Shells and Plates. Cambridge University Press, New York.

[6] Pellicano, F., Amabili, M., and Paidoussis M.P., 2002. "Effect of the geometry on the non-linear vibration of circular cylindrical shells". International Journal of Nonlinear Mechanics, 37, pp. 1181-1198.

[7] Pellicano, F., 2007. "Vibrations of circular cylindrical shells: Theory and experiments". Journal of Sound and Vibration, 303, pp. 154-170. 